\title{
PKD3 promotes metastasis and growth of oral squamous cell carcinoma through positive feedback regulation with PD-L1 and activation of ERK -STAT1/3-EMT signaling
}

\section{Bomiao Cui}

Sichuan University West China College of Stomatology

Jiao Chen

Sichuan University West China College of Stomatology

Min Luo

Sichuan University West China College of Stomatology

Yiying Liu

Sichuan University West China College of Stomatology

Hongli Chen

Sichuan University West China College of Stomatology

Die Lv

Sichuan University West China College of Stomatology

Liwei Wang

Sichuan University West China College of Stomatology

Yingzhu Kang

Sichuan University West China College of Stomatology

\section{Yun Feng}

Sichuan University West China College of Stomatology

Libin Huang

Sichuan University West China Hospital

Ping Zhang ( $\nabla$ pingzhang68@hotmail.com )

\section{Research}

Keywords: OSCC, PKD3, PD-L1, EMT, tumor growth, metastasis

Posted Date: May 14th, 2020

DOl: https://doi.org/10.21203/rs.3.rs-28178/v1 
License: (c) (i) This work is licensed under a Creative Commons Attribution 4.0 International License. Read Full License

Version of Record: A version of this preprint was published at International Journal of Oral Science on March 10th, 2021. See the published version at https://doi.org/10.1038/s41368-021-00112-w. 


\title{
PKD3 promotes metastasis and growth of oral squamous cell carcinoma through positive feedback regulation with PD-L1 and activation of ERK -STAT1/3-EMT signaling
}

Bomiao Cui ${ }^{1 \dagger}$, Jiao Chen ${ }^{1 \dagger}$, Min Luo ${ }^{1 \dagger}$, Yiying Liu ${ }^{1}$, Hongli Chen ${ }^{1}$, Die Lv ${ }^{1}$, Liwei Wang $^{1}$, Yingzhu Kang ${ }^{1}$, Yun Feng ${ }^{1}$, Libin Huang ${ }^{2 *}$, Ping Zhang ${ }^{1 *}$

* Correspondence: huanglibin525@163.com; pingzhang68@hotmail.com

$\dagger^{\dagger}$ Equal contributors

${ }^{1}$ State Key Laboratory of Oral Diseases, West China School of Stomatology, Sichuan University, 14 Renmin South Road Section 3, Chengdu, Sichuan 610041, and P.R. China

${ }^{2}$ Department of Gastroenterology, West China Hospital, Sichuan University, Guo xue Alley 37, Chengdu, Sichuan 610041, and P.R. China

\begin{abstract}
:
Background: Oral squamous cell carcinoma (OSCC) has a high incidence of metastasis. Tumor immunotherapy targeting programmed death ligand 1 (PD-L1) or programmed cell death protein 1 (PD-1) is revolutionary; however, only a few patients with OSCC respond to this treatment. Therefore, it is necessary to understand the molecular mechanisms of OSCC growth and metastasis to identify new therapeutic targets.
\end{abstract}

Methods: In this study, we first analyzed the expression level of protein kinase D3 (PKD3) and PDL1, and their correlation with mesenchymal and epithelial markers through UALCAN database, UCSC Xena, western blot, and immunostaining of human OSCC tissue sections. We knocked down and overexpressed PKD3 in OSCC cell lines and analyzed cell growth, migration, and invasion using western blot, cell proliferation assays, wound healing assays, and Transwell assays. A mouse footpad xenograft model was used to study the effects of PKD3 on tumor growth and lymph node metastasis in vivo.

Results: The expression of PKD3 and PD-L1 in OSCC cells and tissues was significantly increased, and correlated positively with mesenchymal markers but negatively with epithelial markers. Silencing PKD3 significantly inhibited the growth, metastasis, and invasion of OSCC cells, while its overexpression promoted these processes. PKD3 regulated PD-L1 expression through the ERK/STAT1/3 signaling pathway, forming a positive feedback regulatory loop with PD-L1 to induce epithelial-mesenchymal transition (EMT) in OSCC. 
Conclusions: There is a positive feedback regulation between PKD3 and PD-L1, which can drive the EMT of OSCC cells through the ERK/STAT 1/3 pathway, thereby promoting tumor growth and metastasis. Our findings provide a theoretical basis for targeting PKD3 as an alternative method to block EMT for regulating PD-L1 expression and inhibiting OSCC growth and metastasis.

Keywords: OSCC, PKD3, PD-L1, EMT, tumor growth, metastasis

\section{Background}

Head and neck squamous cell carcinoma (HNSCC) is the most common malignant tumor of the head and neck, the most prevalent type of which is oral squamous cell carcinoma (OSCC). OSCC is characterized by aggressive invasion, early cervical lymph node metastasis, high metastasis rate, and fast growth[1,2]. Treatment of patients with metastatic OSCC remains a major clinical challenge. Despite the recent breakthroughs in tumor therapy research, the five-year survival rate of OSCC patients remains approximately 50\%[3]. Many tumor patients nowadays receive immunotherapy, which has also been approved for the treatment of recurrent or metastatic HNSCC. Notwithstanding some success, only a few patients respond to the treatment. Therefore, understanding the mechanisms underlying the growth and metastasis of OSCC is crucial in order to develop new treatment strategies.

The epithelial-mesenchymal transition (EMT) is one of the critical processes that promote metastasis[4, 5]. In the course of EMT, epithelial cells lose polarity, recombine actin cytoskeleton, and acquire more mesenchymal characteristics, all processes which enhance their metastatic ability. EMT is characterized by reduced expression of adhesion junction proteins (such as E-cadherin) and increased expression of mesenchymal markers (such as N-cadherin, Snail, and Vimentin) through transcriptional and post-transcriptional mechanisms[5-7]. Moreover, Snail can regulate the transcriptional repression of the epithelial marker E-cadherin (CDH1) during EMT[8]. Hence, identifying potential EMT blockers in patients with OSCC may pave the way for promising new therapies.

Protein kinase D (PKD) are a class of serine/threonine protein kinases that modulate numerous biological activities, such as protein transport, cell migration, differentiation, proliferation, 
apoptosis, EMT, and immune regulation[9-15]. PKD3 expression is increased in highly invasive breast and prostate cancers[9-15]. The concomitant absence of PKD1 in highly invasive breast cancer indicates that, while this protein plays an anti-tumor role, PKD3 may instead promote cancer. Differently from PKD1 and PKD3, the expression of PKD2 during breast cancer progression is relatively unchanged[13, 15, 16]. Moreover, the downregulation of PKD3 has a more significant effect on cell migration than that of PKD2[12]. Our research group obtained similar results in OSCC, and found a significant correlation between PKD3 nuclear localization and advanced tumor grade[14]. Increasing evidence shows that PKD3 is involved in multiple oncogenic signaling pathways, including extracellular signal-regulated kinase 1/2 (ERK1/2), protein kinase B (AKT), nuclear factor-kappa $\mathrm{B}(\mathrm{NF}-\mathrm{kB})$, and signal transducer and activator of transcription $1 / 3$ $(\mathrm{STAT} 1 / 3)[12,13,17]$. These pathways can regulate the EMT as well as tumor cell growth and metastasis[18-22]. EMT may also contribute to the immune escape of tumor cells[23]. ERK1/2, NF$\mathrm{\kappa B}$, and STAT1/3 are also important regulators of PD-L1 expression[24].

Previously, we showed that PKD3 can regulate the expression of PD-L1. In this study, we investigated whether PKD3 participates in EMT of OSCC, and examined the contribution of PDL1 and signaling pathways to induction of the EMT phenotype.

\section{Materials and Methods}

\section{Cell culture}

Human normal oral epithelial keratinocytes $(\mathrm{HOK})$ were cultured in keratinocyte serum-free medium (KSFM) with bovine pituitary extract $(50 \mu \mathrm{g} / \mathrm{ml})$ and recombinant human epidermal growth factor (5 ng/ml) (Gibco, USA). SCC25 cells were cultured in DMEM/F12 medium (Gibco, USA) containing 10\% Fetal Bovine Serum (FBS; PAN, Germany), 400 ng/ml hydrocortisone, 100 $\mathrm{IU} / \mathrm{ml}$ penicillin, and $100 \mu \mathrm{g} / \mathrm{ml}$ streptomycin (HyClone, Logan, UT, United States). Dysplastic oral keratinocytes (DOK) and three other oral squamous cell lines (Cal-27, HSC-4, and HSC-3) were cultured in DMEM/HIGH GLUCOSE medium containing 10\% FBS, $100 \mathrm{IU} / \mathrm{ml}$ penicillin, and 100 $\mu \mathrm{g} / \mathrm{ml}$ streptomycin. The culture medium for DOK also contained $5 \mu \mathrm{g} / \mathrm{ml}$ hydrocortisone. HOK were purchased from ScienCell Research Laboratories, Inc. DOK were supplied by the European Collection of Authenticated Cell Cultures (ECACC). The four OSCC cell lines (Cal-27, HSC-4, 
SCC25, and HSC-3) were obtained from the American Type Culture Collection (ATCC). All cells were maintained at $37^{\circ} \mathrm{C}$ in a humidified atmosphere with $5 \% \mathrm{CO}_{2}$.

\section{Plasmid and siRNA transfection}

CSHCTR001-LVRU6GP-control and HSH006319-LVRU6GP-PKD3 (clone ID NM_005813.3) shRNA plasmids were purchased from GeneCopoeia. The target sequences are listed in Table S1. Specific siRNAs against STAT1, STAT3, ERK1, ERK2, and si-control were synthesized by Genepharma (Shanghai, China) (Table S2). Specific siRNAs against PKD3 and PD-L1 were purchased from Invitrogen. Lipofectamine 2000 reagent was used to transfect sh-RNA plasmids into Cal-27 and HSC-4, and si-RNA into Cal-27 according to the manufacturer's protocol. Puromycin $(0.2 \mu \mathrm{g} / \mathrm{ml})$ was used to screen Cal-27 and HSC-4 cell lines stably silencing PKD3. DOK and Cal27 cell lines stably overexpressing PKD3 were generated similarly. Human PKD3-overexpressing DOK and Cal-27 cell lines were established using pEX-U1233-M02 (control: pEX-EGFP-M02; GeneCopoeia).

\section{Patients and clinical samples}

In this study, a total of 34 patients with OSCC who did not receive radiotherapy or chemotherapy before surgery were enrolled, and 26 non-tumor oral epithelial tissues were collected from West China Hospital of Stomatology, Sichuan University. The patients' clinical information is shown in Table S3. All clinical samples were confirmed by pathological examination. This study was approved by the Ethics Committee of West China Hospital of Stomatology, Sichuan University, and all patients gave informed consent.

\section{Western blot}

Total proteins were extracted for western blot detection according to the method described previously[14]. Protein concentration was measured using the BCA protein assay kit (Beyotime Institute of Biotechnology, China). Approximately $20 \mu \mathrm{g}$ of protein was separated using $6 \%$ or $10 \%$ SDS-PAGE, and then transferred to PVDF membrane (Bio-Rad, USA). After blocking the PVDF membrane in $5 \%$ skim milk at room temperature for $1 \mathrm{~h}$, the primary antibody was added and incubated overnight on a shaker at $4^{\circ} \mathrm{C}$. Membranes were washed with TBST and then incubated with horseradish peroxidase (HRP)-conjugated anti-mouse or anti-rabbit secondary antibodies (cat. 
no. 7076 or 7074; 1:2,000; Cell Signaling Technology, Inc.) for 1-2 $\mathrm{h}$ at room temperature. An enhanced chemiluminescence (ECL) substrate kit (Millipore, Inc.) and an automatic gel imaging system (Bio-Rad Laboratories, Inc.) were used to detect the luminescent signal of protein bands. The primary antibodies used for western blot were the following: PKD3 (cat. no. 5655; 1:1,000), PD-L1 (cat. no. 13684; 1:1,000), E-cadherin (cat. no. 3195; 1:1,000), N-cadherin (cat. no. 13116; 1:1,000), Vimentin (cat. no. 5741; 1:1,000), Snail (cat. no. 3879; 1:1,000), ERK1/2 (cat. no. 4696; 1:1,000), and phospho-p44/42 MAPK (Erk1/2) (Thr202/Tyr204) (cat. no. 4370; 1:1,000) from Cell Signaling Technology; and STAT1 (cat. no. ab109320; 1:10,000), phospho-STAT1(S727) (cat. no. ab109461; 1:5,000), phospho-STAT1(Y701) (cat. no. ab29045; 1:1,000), STAT3 (cat. no. ab68153; 1:2,000), phospho-STAT3(S727) (cat. no. ab32143; 1:5,000), phospho-STAT3(Y705) (cat. no. ab76315; 1:10,000), NF-kB P65 (cat. no. ab32536; 1:20,000), phospho-NF-кB P65 (cat. no. ab109458; 1:5,000), and GAPDH (cat. no. ab128915; 1:20,000) from Abcam. Phos-tag SDS-PAGE (Wako) was performed according to the protocol described previously[25]. The densitometry of protein bands was quantified using Gel-Pro 32 software (version 3.1, Media Cybernetics, Bethesda, MD, USA).

\section{Immunohistochemical staining}

All excised OSCC tissues and lymph nodes of mice were fixed with $10 \%$ neutral formalin, embedded in paraffin, and cut into 5 - $\mu$ m-thick sections. Immunohistochemical staining was performed as previously described[14]. The antibodies used for this method include PKD3 (cat. No. ab252982; 1:40; Abcam), and PD-L1 (cat. No. 13684; 1:200), E-cadherin (cat. no. 3195; 1:400; ), and Vimentin (cat. no. 5741; 1:200) from Cell Signaling Technology.

\section{Colony formation and cell counting Kit-8 assay}

After transfection with PKD3 shRNA or PKD3-overexpression plasmid, cells in the logarithmic growth phase were harvested, dissociated with trypsin, washed twice with PBS buffer, and resuspended with DMEM/HIGH GLUCOSE medium containing 10\% FBS. The cell suspension was diluted in a gradient manner, and 500 cells were inoculated in a 6-cm petri dish containing $3 \mathrm{ml}$ of medium preheated at $37^{\circ} \mathrm{C}$. Then, the cells were gently inoculated to evenly disperse. Thereafter, the medium was replaced every 3 days. After 2 weeks, the medium was removed, and the colonies 
were stained with $0.1 \%$ crystal violet for $15-30 \mathrm{~min}$. The petri dishes were scanned, and the number of clones in each dish was calculated using Image J $1.48 \mathrm{v}$ software.

After gradient dilution of the cell suspension, the cells were seeded at a density of $3 \times 10^{3}$ cells per well in 96-well plates (100 $\mu \mathrm{l} /$ well), and at least three replicate wells were set per group. After $24 \mathrm{~h}$, Cell Counting Kit-8 (CCK-8) was used to detect cell proliferation (DOJINDO, Japan). The test was continued for 6 days, with daily incubation time of $1 \mathrm{~h}$. The growth curve was made according to the OD value at $450 \mathrm{~nm}$.

\section{Cell invasion and migration assay}

Cell invasion assays were performed using 24-well Transwell chambers $(8-\mu \mathrm{m}$ pore size; BD Bioscience, San Jose, CA, USA). Cells $\left(1 \times 10^{5}\right)$ were seeded in the upper chamber, which was coated with $50 \mu 1$ Matrigel ( $5 \mathrm{mg} / \mathrm{ml}$ in cold medium, BD Bioscience, Franklin Lakes, NJ, USA) and cultured with $1 \%$ FBS. Then, $600 \mu \mathrm{l}$ DMEM/HIGH GLUCOSE medium supplemented with $10 \%$ FBS was added to the lower chamber. After incubation at $37^{\circ} \mathrm{C}$ for $24 \mathrm{~h}$, the cells were fixed with 4\% paraformaldehyde for $15 \mathrm{~min}$. The cells and Matrigel in the upper chamber were removed and washed with PBS three times, and the invading cells were stained with $0.1 \%$ crystal violet. The chamber membrane was placed on a glass slide and 10 fields of view were randomly selected under the microscope to take pictures and count stained cells. Image J $1.48 \mathrm{v}$ software was used to count the number of cells. Each group had 3 repetitions.

Cell migration assays were performed similarly to cell invasion assays, but without Matrigel in the upper chamber. The same methods described above were used to fix, stain, photograph, and count the cells.

\section{The Cancer Genome Atlas (TCGA) data collection and analysis}

UALCAN database (http://ualcan.path.uab.edu/analysis.html) was used to analyze the gene expression levels of PKD3, PD-L1, and EMT markers. UCSC Xena (http://xena.ucsc.edu/welcometo-ucsc-xena/) was used to analyze the correlation of the expression levels of query genes in 564 HNSCC patients. The correlation heat map was made using Origin2018 software (OriginLab, Northampton, MA, USA). Spearman's $r$ test was used for correlation analysis of TCGA data.

\section{Popliteal lymph node metastasis model}


The animal experiments were approved by the Ethics Review Committee of West China Hospital of Stomatology, Sichuan University (permit number: WCHSIRB-D-2017-211). Female BALB/c-nu mice (4-5 weeks old) were purchased from the Transgenic Engineering Mouse Center of West China Hospital of Sichuan University. The mice were randomly divided into 3 groups (Con, sh-PKD3-1, and sh-PKD3-2), with 9 mice per group. All transfected cells stably expressed green fluorescent protein (GFP). Cal-27 cells $\left(4 \times 10^{6} /\right.$ mice $)$ were injected subcutaneously into the footpad of the left hind leg. Four weeks later, the nude mice were euthanized. Primary tumors and popliteal lymph nodes were removed. The tissues were fixed with $10 \%$ neutral formalin and paraffin embedded. Serial $5-\mu \mathrm{m}$-thick slices were cut and analyzed by immunohistochemistry (IHC) with rabbit antihuman PKD3 polyclonal antibody (cat. no. ab252982; 1:40; Abcam), rabbit anti-human PD-L1 polyclonal antibody (cat. no. 13684; 1:200; Cell Signaling Technology), or rabbit anti-GFP monoclonal antibody (cat. no. ab183734; 1:200; Abcam).

\section{Statistical analysis}

Correlation analysis was performed using Origin 2018 software (OriginLab). Other analyses were performed using GraphPad Prism software (version 6; GraphPad Software, Inc.) using one-way ANOVA followed by Tukey's multiple comparison test. The $\chi^{2}$ test was used to analyze the relationship between PKD3 expression and lymph node metastasis. $\mathrm{P}<0.05$ was considered statistically significant.

\section{Results}

\section{PKD3 is overexpressed in OSCC and is closely related to EMT}

Our previous studies determined that PKD3 can regulate PD-L1 expression[14]. Moreover, PD-L1 is closely related to EMT in HNSCC[26]. To verify whether PKD3 is involved in the regulation of EMT in OSCC, we first analyzed the expression levels of PKD3 and PD-L1 in normal and HNSCC tissues using the UALCAN database. As shown in Fig. 1a, the levels of PKD3 and PD-L1 were significantly increased in tumor tissues. We downloaded the RNAseq data of the query genes from UCSC Xena and obtained the gene expression data of 564 HNSCC specimens to analyze the correlation between the expression of PKD3, PD-L1, and EMT-related markers. In this large dataset, the expression of PKD3 and PD-L1 was positively correlated with mesenchymal markers (such as 
SNAI1, SNAI2, ZEB1, ZEB2, VIM) and negatively correlated with epithelial markers (such as CDH1, CLDN4, CLDN7) (Fig. 1b). Subsequently, we analyzed the protein levels of PKD3, PD-L1, and EMT markers in OSCC cell lines and tissues using western blot and IHC. As shown in Fig. 1c and d, PKD3 was not only highly expressed in OSCC cell lines, but also positively correlated with mesenchymal markers (N-cadherin, Snail, Vimentin) but negatively correlated with epithelial markers (E-cadherin). Moreover, the levels of PKD3, PD-L1, and Vimentin were higher in OSCC tissues than in normal tissues. With advanced tumor grade, the nuclear distribution of PKD3 as well as the levels of PKD3 and Vimentin gradually increased (Fig. 1e and f). However, the expression of PKD3 did not correlate with either age, sex, or race (Figure S1). In summary, our data indicate that PKD3 may play an important role in the occurrence and development of OSCC and is closely related to the EMT of OSCC.

\section{Knockdown of PKD3 significantly inhibits growth, migration, and invasion of OSCC cells}

To determine its role in the EMT of OSCC, we silenced the expression of PKD3 in the OSCC cell lines Cad-27 (without obvious mesenchymal characteristics) and HSC-4 (with obvious mesenchymal characteristics), considering the results of Fig. 1c. We employed sh-PKD3-1 and shPKD3-2 as we previously determined that these two shRNAs can effectively knock down PKD3. First, we analyzed the effect of PKD3 on cell proliferation using clone formation and CCK-8 assay. After 2 weeks, the clone formation rate of PKD3-silenced cells was significantly reduced (Fig. 2a). CCK-8 assay showed that the growth of PKD3 knockdown cells was much slower than that of the control cells. Additionally, statistical analysis showed significant differences from the third day (Fig. 2b). Next, we evaluated the effect of PKD3 on the invasion and metastasis of OSCC cells. Transwell migration assay showed that PKD3 knockdown cells migrated more slowly than control cells (Fig. 2c). Moreover, knockdown of PKD3 significantly inhibited wound healing of Cal-27 and HSC-4 cells (Fig. 2d). PKD3 silencing also significantly reduced the invasion ability of OSCC cells through Matrigel membrane (Fig. 2e). Further, we examined the effects of PKD3 silencing on epithelial and mesenchymal markers using western blot. As shown in Fig. 2f, expression of E-cadherin was increased while that of mesenchymal markers and PD-L1 was decreased in PKD3 knockdown cells compared to wild-type and control cells. Together, these results implicate that knockout of PKD3 inhibits the migration, invasion, and EMT phenotype of OSCC cells. 


\section{Overexpression of PKD3 promotes growth, migration and invasion of OSCC cells}

According to the results of Fig. 1c, we selected two cell lines with lower PKD3 expression, namely DOK and Cal-27. After screening, we obtained cells stably overexpressing PKD3 and evaluated their proliferation, invasion, and migration. We found that the number of clones formed by PKD3overexpressing cells increased significantly (Fig. 3a). Growth curve analysis showed that PKD3overexpressing cells also proliferated much faster than control cells. DOK cells showed statistical differences from the fourth day, while Cal-27 showed significant differences from the third day (Fig. 3b). Transwell and wound healing assays showed that PKD3 overexpression significantly promoted the migration and invasion of DOK and Cal-27 (Fig. 3c-e). Western blot analysis revealed that, in PKD3-overexpressing cells, the expression of PD-L1 slightly increased, while the expression of epithelial and mesenchymal markers decreased and increased, respectively (Fig. 3f). Altogether, these data demonstrate that PKD3 contributes to regulating the EMT of OSCC, and that its overexpression promotes the EMT phenotype and metastasis of OSCC.

\section{PKD3 plays an important role in the EMT of OSCC induced by the PD-1/PD-L1 pathway}

Programmed death ligand-1 (PD-L1) can interact with programmed cell death protein 1 (PD-1) expressed on activated B cells, T cells, and natural killer cells, thus activating PD-1/PD-L1 pathway, leading to $\mathrm{T}$ cell apoptosis or loss of function, and downregulating $\mathrm{T}$ cell anti-tumor response[27]. But how does the activation of the PD-1/PD-L1 pathway affect tumor cells? To address this problem, we stimulated Cal-27 cells with different concentrations of PD-1 fusion protein for $24 \mathrm{~h}$. As shown in Fig. $4 \mathrm{a}$ and $\mathrm{b}$, cells treated with 1, 2, and $5 \mu \mathrm{g} / \mathrm{ml}$ PD-1 fusion protein showed a slight decrease in E-cadherin expression and a significant increase in PKD3 and PD-L1 expression compared to cells treated with low concentration $(0.5 \mu \mathrm{g} / \mathrm{ml})$ of fusion protein or untreated. We then examined the activation of PKD3 using Phos-tag SDS-PAGE immunoblot. PKD3 phosphorylation began to increase significantly upon treatment with $1 \mu \mathrm{g} / \mathrm{ml}$ PD-1. Intriguingly, the stimulation of cells with all four tested concentrations of PD-1 significantly upregulated N-cadherin, Snail, and Vimentin. Moreover, the levels of Snail and Vimentin augmented gradually with increasing concentrations of PD-1 fusion protein. These data suggest that the binding of PD-1 fusion protein to PD-L1 can promote the EMT of OSCC.

Subsequently, we investigated whether PD-1/PD-L1 pathway-induced EMT in OSCC was 
dependent on PKD3. For this purpose, we examined the expression of epithelial and mesenchymal markers after $24 \mathrm{~h}$ of treatment with or without $1 \mu \mathrm{g} / \mathrm{ml}$ PD-1 fusion protein. The expression of PDL1, N-cadherin, Snail, and Vimentin was increased upon treatment with PD-1; however, knockdown of PKD3 significantly reduced the expression of PD-L1 and mesenchymal markers, but increased the expression of the epithelial marker E-cadherin (Fig. 4c and d). Taken together, these results show that knockdown of PKD3 can effectively block the EMT induced by the PD-1/PD-L1 pathway.

\section{PKD3 regulates EMT and PD-L1 through ERK1/2 and STAT1/3 signaling, and establishes a positive feedback loop with PD-L1}

To further explore the mechanism of PKD3-induced OSCC migration and tumor growth, we silenced PKD3 and PD-L1 in Cal-27 cells using siRNAs. As shown in Fig. 5a and b, the expression of PD-L1 was significantly reduced in PKD3-knockdown cells. In these cells, the levels of EMT markers showed the same changes previously observed in sh-PKD3-transfected cells. Additionally, knockdown of PKD3 significantly inhibited the phosphorylation of STAT1/3 at Ser727, but did not significantly decrease the phosphorylation of tyrosine residues. Phosphorylation of ERK1/2 was also significantly reduced after PKD3 knockdown. Considering that PKD3 could regulate the expression of PD-L1, which is closely associated with EMT, we speculated that the regulation of EMT by PD-L1 might also involve the ERK/STAT signaling pathways. Interestingly, knockdown of PD-L1 also reduced PKD3 expression. Following PD-L1 knockdown, expression of mesenchymal markers was significantly reduced, while expression of E-cadherin was slightly increased. Furthermore, the knockdown of PD-L1 significantly reduced phosphorylation of STAT1/3 at serine residues, as well as phosphorylation, but not expression, of ERK1/2. Double knockdown of PKD3 and PD-L1 inhibited the phosphorylation of ERK1/2 and STAT1/3 on serine residues more effectively than single knockdown of either gene (Fig. 5c). In addition, the double knockdown of PKD3 and PD-L1 significantly inhibited the EMT of OSCC, similarly to the single knockdown of PKD3. However, knockdown of neither PKD3 nor PD-L1 could significantly alter the NF- $\kappa \mathrm{B}$ pathway.

As ERK1/2 and STAT1/3 play a crucial role in EMT, we analyzed their effect on the EMT of OSCC cells. After knocking down ERK1/2 and STAT1/3, the expression of PKD3 and PD-L1 was effectively suppressed in Cal-27 cells. Moreover, upon silencing of ERK1/2 and STAT1/3, 
mesenchymal markers in OSCC cell lines were still significantly reduced, even if stimulated with PD-1 fusion protein, while E-cadherin was only slightly affected (Fig. 6a, b and c). Furthermore, the PD-1/PD-L1 interaction significantly increased the phosphorylation of ERK1/2 and STAT1/3; however, the phosphorylation of STAT1/3 at S727 site was significantly inhibited after ERK1/2 knockdown (Fig. 6d). In conclusion, PKD3 and PD-L1 regulate the phosphorylation levels of ERK1/2 and STAT1/3. Interestingly, ERK1/2 and STAT1/3 in turn can also affect the expression of PKD3 and PD-L1, and thereby regulate the EMT of OSCC. These data indicate that PKD3 regulates PD-L1 expression and EMT of OSCC through ERK1/2 and STAT1/3, suggesting a positive feedback regulation between them.

\section{PKD3 promotes the growth and lymph node metastasis of OSCC in vivo}

To study the effects of PKD3 on the growth and lymph node metastasis of OSCC in vivo, we injected Cal-27-sh-Con, Cal-27-sh-PKD3-1, or Cal-27-sh-PKD3-2 cells into the mouse footpad to establish a xenograft model (Fig. 7a). After 4 weeks, the mice were sacrificed, the size of the footpad tumors was measured, and the popliteal lymph nodes were removed and measured. Tumors and lymph nodes showed the largest size in the Cal-27-sh-Con group, and were smaller in the Cal-27-sh-PKD31 and Cal-27-sh-PKD3-2 groups (Fig. 7b and d). Moreover, IHC staining revealed that the expression of PD-L1 decreased with the knockdown of PKD3 (Fig. 7c). Since the lentiviral plasmids are labeled with GFP, transfected cancer cells that metastasized into lymph nodes could be identified by IHC staining of GFP (Fig. 7e). Subsequently, we calculated the lymph node metastasis rates in different groups. The lymph node metastasis rate was highest in the Cal-27-sh-Con group (78\%), followed by Cal-27-sh-PKD3-1 (22\%), and Cal-27-sh-PKD3-2 (11 \%) (Fig. 7e). Importantly, the observed difference in metastasis rate between the control group and the two PKD3 knockdown groups was significant, as determined by $\chi^{2}$ test $(\mathrm{P}<0.05$, Fig. $7 \mathrm{e})$. Our experiment thus proved that PKD3 can promote the growth and metastasis of OSCC in vivo.

\section{Discussion}

OSCC is a common malignant tumor of the head and neck characterized by rapid growth and high metastasis rate. Increasing evidence suggests that EMT is crucial for tumor progression and metastasis. In the initial stages of metastasis, epithelial tumor cells undergo EMT, leading to loss of 
intercellular adhesion, enhanced mobility, and invasion of surrounding tissues[5, 28]. Currently, the treatment of OSCC patients with metastasis remains a major clinical challenge. Therefore, it is urgent to explore the potential mechanism of growth and metastasis of OSCC to determine novel and more effective therapeutic targets.

In this study, we investigated the relevance of PKD3, a poorly characterized member of the PKD family, to OSCC. Our research revealed that PKD3 regulates EMT and PD-L1 expression in OSCC through ERK1/2 and STAT1/3 pathways, and establishes a positive feedback loop with PD-L1 to promote tumor growth and metastasis. These findings indicate that PKD3 plays a pivotal role in the progression of OSCC, suggesting PKDs as potential therapeutic targets for treatment of this type of cancer.

Based on data from the UALCAN database, UCSC Xena, and western blot, PKD3 and PD-L1 are significantly upregulated in OSCC. Previous studies showed that PD-L1 regulates EMT and promotes tumor growth and metastasis[23, 26, 29-33]. Moreover, PD-L1 expression can be modulated by PKD3[14]. Although this evidence implies that PKD3 may be closely related to EMT, no molecular mechanism has previously been reported between the two. Here, we found that PKD3 was positively correlated with mesenchymal markers and negatively correlated with epithelial markers. Downregulation of PKD3 inhibited the invasion, migration, and proliferation of OSCC in vitro; reduced tumor growth and metastasis in vivo; and decreased the expression of mesenchymal markers while increasing that of E-cadherin. PKD3 overexpression instead significantly enhanced the ability of OSCC cells to invade, migrate, and proliferate, promoted expression of mesenchymal markers, and inhibited that of E-cadherin. IHC and TCGA analysis showed that the expression of PKD3, the frequency and intensity of its nuclear staining, as well as the expression of Vimentin gradually increased with the increase of tumor grade. These results support our view that PKD3 can regulate EMT in OSCC and promote tumor growth and metastasis.

PD-L1 interacts with PD-1 expressed by activated T cells, B cells, natural killer (NK) cells, some dendritic cells (DC), and tumor-associated macrophages, activating the PD-1/PD-L1 pathway. In turn, activation of this pathway can inhibit the anti-tumor function of the same immune cells, thereby reducing anti-tumor immunity[27, 34-37]. However, the effect of PD-1/PD-L1 interaction on tumor 
cells has rarely been reported. Here, we found that the expression of PKD3 and PD-L1 increased significantly after treatment of OSCC cell lines with the PD-1 fusion protein; in parallel, the expression of mesenchymal markers increased, and that of epithelial markers decreased, suggesting that the PD-1/PD-L1 pathway can induce EMT in tumor cells. EMT is key to tumor cell remodeling for metastasis[5]. The interaction between tumor cells and tumor-infiltrating lymphocytes induces the expression of PD-L1 in tumor cells through the STAT pathway; in HNSCC, the expression of PD-L1 is closely related to EMT $[26,38,39]$. These results are consistent with ours, which indicate that, in vivo, PD-1-expressing T cells, B cells, and natural killer cells interact with OSCC cells, promoting their EMT and immune escape. This may also be the cause of early lymph node metastasis in OSCC.

The nuclear localization and catalytic activation of PKD3 are linked to the activation of G proteincoupled receptor signaling pathways[40], which include STAT1/3, ERK, and NF-kB[41-44]. These pathways, which are also regulated by PKD3, influence cell growth, metastasis, EMT, and other biological processes, but also modulate the expression of PD-L1[12-14, 17-22, 24]. The synchronicity of these different biological processes suggests the possibility of a common genetic drive. In OSCC cells, silencing PKD3 can effectively block the EMT induced by PD-1/PD-L1 interaction. Transient knockdown of ERK1/2, STAT1, and STAT3 also abolishes PD-1/PD-L1induced EMT. As PKD3 mainly induces activation of ERK1/2 and STAT1/3, we concluded that PKD3 may regulate EMT in OSCC cells through ERK1/2 and STAT1/3. Interestingly, we found that knockdown of PKD3 reduced the expression of PD-L1, and conversely expression of PKD3 also decreased after knocking down PD-L1. Moreover, PD-1 fusion protein induced the expression of PD-L1 and PKD3. Knockdown of PD-L1 could also affect the activation of ERK1/2 and STAT1/3. PD-L1 was previously shown to regulate the activation of ERK1/2 by binding with RAS, and consequently affect the EMT of tumor cells[33]. Additionally, the activation of STAT1/3 can be mediated by ERK1/2[45-51]. These findings are consistent with our results, which suggest that there is a positive feedback loop between PKD3 and PD-L1 that drives EMT through the ERK/STAT1/3 signal, ultimately promoting tumor growth and metastasis (Fig. 8).

\section{Conclusions}


In conclusion, we determined that PD-1/PD-L1 interaction could promote EMT in OSCC. Further, we demonstrated that PKD3 regulates PD-L1 expression through the ERK/STAT1/3 pathway, and that there is a positive feedback loop between PKD3 and PD-L1 driving EMT and promoting the growth and metastasis of OSCC. Our study thus suggests that targeting PKD3 could be an effective strategy to block EMT, providing a new potential avenue for treatment of OSCC.

\begin{abstract}
Abbreviations
PKD: Protein kinase D; OSCC: Oral squamous cell carcinoma; HNSCC: Head and neck squamous cell carcinoma; PD-L1: Programmed death ligand 1; PD-1: Programmed cell death protein 1; TCGA: The Cancer Genome Atlas; EMT: Epithelial-mesenchymal transition; ERK1/2: Extracellular signalregulated kinase; AKT: Protein kinase B; STAT: Signal transducer and activator of transcription; NF-kB: Nuclear factor-kappa B; HOK: Human normal oral epithelial keratinocytes; KSFM: Keratinocytes serum-free medium; FBS: Foetal bovine serum; DOK: Dysplastic oral keratinocyte cell line; ECACC: European Collection of Authenticated Cell Cultures; ATCC: American Type Culture Collection
\end{abstract}

\title{
Acknowledgements
}

We would like to thank the patients and researchers involved in this study.

\section{Authors contributions}

PZ, LBH and YF designed the outline of the study. BMC, JC and ML designed the study, conducted experiments and wrote the manuscript. JC and ML supervised the study, contributed to data acquisition and revised the manuscript. BMC, YYL, HLC, DL, LWW and YZK collected and analyzed the data. All authors read and approved the final manuscript.

\section{Funding}

This study was supported by National Natural Science Foundations of China (grant nos. 81802717 and 81372892).

\section{Availability of data and materials}

All data generated or analysed during this study are included in this published article and its supplementary information files.

\section{Ethics approval and consent to participate}

All procedures of human and mouse experiments were approved by the Ethics Review Committee 
of West China hospital of Stomatology, Sichuan University (permit number: WCHSIRB-D-2017211).

\section{Consent for publication}

All authors agree to publish this article.

\section{Competing interests}

The authors declare that they have no conflict of interest.

\section{References}

1. Torre LA, Bray F, Siegel RL, Ferlay J, Lortet-Tieulent J, Jemal A. Global cancer statistics, 2012. CA: A Cancer Journal for Clinicians. 2015;65.

2. Sathiyasekar AC, Chandrasekar P, Pakash A, Kumar KG, Jaishlal M. Overview of immunology of oral squamous cell carcinoma. Journal of pharmacy bioallied sciences. 2016;8:S8.

3. Carreras-Torras C, Gay-Escoda C. Techniques for early diagnosis of oral squamous cell carcinoma: Systematic review. Medicina Oral Patologia Oral Y Cirugia Bucal. 2015;20:e305.

4. Chaffer CL, Weinberg RA. A perspective on cancer cell metastasis. science. 2011;331:155964.

5. Heerboth S, Housman G, Leary M, Longacre M, Byler S, Lapinska K, et al. EMT and tumor metastasis. Clinical translational medicine. 2015;4:6.

6. Thiery JP. Epithelial-mesenchymal transitions in tumour progression. Nature Reviews Cancer. $2002 ; 2: 442-54$

7. Lamouille S, Xu J, Derynck R. Molecular mechanisms of epithelial-mesenchymal transition. Nature reviews Molecular cell biology. 2014;15:178.

8. Cano A, Pérez-Moreno MA, Rodrigo I, Locascio A, Blanco MJ, del Barrio MG, et al. The transcription factor snail controls epithelial-mesenchymal transitions by repressing E-cadherin expression. Nature cell biology. 2000;2:76-83.

9. Roy A, Ye J, Deng F, Wang QJ. Protein kinase D signaling in cancer: a friend or foe? Biochimica et Biophysica Acta. 2017;1868:283-94.

10. Durand N, Borges S, Storz P. Protein kinase D enzymes as regulators of EMT and cancer cell invasion. Journal of clinical medicine. 2016;5:20.

11. Chen J, Feng Y, Lu L, Wang H, Dai L, Li Y, et al. Interferon- $\gamma$-induced PD-L1 surface 
expression on human oral squamous carcinoma via PKD2 signal pathway. Immunobiology. 2012;217:385-93

12. Zou Z, Zeng F, Xu W, Wang C, Ke Z, Wang QJ, et al. PKD2 and PKD3 promote prostate cancer cell invasion by modulating NF-kB-and HDAC1-mediated expression and activation of uPA. J Cell Sci. 2012;125:4800-11.

13. Chen J, Deng F, Singh SV, Wang QJ. Protein kinase D3 (PKD3) contributes to prostate cancer cell growth and survival through a PKCE/PKD3 pathway downstream of Akt and ERK 1/2. Cancer research. 2008;68:3844-53.

14. Cui B, Chen J, Luo M, Wang L, Chen H, Kang Y, et al. Protein kinase D3 regulates the expression of the immunosuppressive protein, PD-L1, through STAT1/STAT3 signaling. International Journal of Oncology. 2020;56:909-20.

15. Huck B, Duss S, Hausser A, Olayioye MA. Elevated protein kinase D3 (PKD3) expression supports proliferation of triple-negative breast cancer cells and contributes to mTORC1-S6K1 pathway activation. Journal of Biological Chemistry. 2014;289:3138-47.

16. Borges S, Perez EA, Thompson EA, Radisky DC, Geiger XJ, Storz P. Effective Targeting of Estrogen Receptor-Negative Breast Cancers with the Protein Kinase D Inhibitor CRT0066101. Molecular cancer therapeutics. 2015;14:1306-16.

17. Baker J, Falconer AM, Wilkinson DJ, Europe-Finner GN, Litherland GJ, Rowan AD. Protein kinase D3 modulates MMP1 and MMP13 expression in human chondrocytes. PloS one. 2018;13.

18. Wang K, Ji W, Yu Y, Li Z, Niu X, Xia W, et al. FGFR1-ERK1/2-SOX2 axis promotes cell proliferation, epithelial-mesenchymal transition, and metastasis in FGFR1-amplified lung cancer. Oncogene. 2018;37:5340-54.

19. Khan S, Shukla S, Sinha S, Lakra AD, Bora HK, Meeran SM. Centchroman suppresses breast cancer metastasis by reversing epithelial-mesenchymal transition via downregulation of HER2/ERK1/2/MMP-9 signaling. The international journal of biochemistry cell biology. 2015;58:1-16.

20. Feng Z, Guo S. Tim-3 facilitates osteosarcoma proliferation and metastasis through the NFkappaB pathway and epithelial-mesenchymal transition. Genet Mol Res. 2016;15:gmr7844.

21. Sun Y, Cheng M-K, RL Griffiths T, Kilian Mellon J, Kai B, Kriajevska M, et al. Inhibition of STAT signalling in bladder cancer by diindolylmethane-relevance to cell adhesion, migration and 
proliferation. Current cancer drug targets. 2013;13:57-68.

22. Cui Y, Li Y-Y, Li J, Zhang H-Y, Wang F, Bai X, et al. STAT3 regulates hypoxia-induced epithelial mesenchymal transition in oesophageal squamous cell cancer. Oncology reports. 2016;36:108-16.

23. Alsuliman A, Colak D, Al-Harazi O, Fitwi H, Tulbah A, Al-Tweigeri T, et al. Bidirectional crosstalk between PD-L1 expression and epithelial to mesenchymal transition: significance in claudin-low breast cancer cells. Molecular cancer. 2015;14:149.

24. Ritprajak P, Azuma M. Intrinsic and extrinsic control of expression of the immunoregulatory molecule PD-L1 in epithelial cells and squamous cell carcinoma. Oral oncology. 2015;51:221-8.

25. W Q, molecular SSJJo, cardiology c. Phos-tag SDS-PAGE resolves agonist- and isoformspecific activation patterns for PKD2 and PKD3 in cardiomyocytes and cardiac fibroblasts. Journal of molecular cellular cardiology. 2016;99:14-22.

26. Ock C-Y, Kim S, Keam B, Kim M, Kim TM, Kim J-H, et al. PD-L1 expression is associated with epithelial-mesenchymal transition in head and neck squamous cell carcinoma. Oncotarget. 2016;7:15901.

27. Dong H, Strome SE, Salomao DR, Tamura H, Hirano F, Flies DB, et al. Tumor-associated B7H1 promotes T-cell apoptosis: a potential mechanism of immune evasion. Nature medicine. 2002;8:793-800.

28. Guarino M, Rubino B, Ballabio G. The role of epithelial-mesenchymal transition in cancer pathology. Pathology. 2007;39:305-18.

29. Chen L, Gibbons DL, Goswami S, Cortez MA, Ahn Y-H, Byers LA, et al. Metastasis is regulated via microRNA-200/ZEB1 axis control of tumour cell PD-L1 expression and intratumoral immunosuppression. Nature communications. 2014;5:1-12.

30. Noman MZ, Janji B, Abdou A, Hasmim M, Terry S, Tan TZ, et al. The immune checkpoint ligand PD-L1 is upregulated in EMT-activated human breast cancer cells by a mechanism involving ZEB-1 and miR-200. OncoImmunology.6:e1263412.

31. Kim S, Koh J, Kim M-Y, Kwon D, Go H, Kim YA, et al. PD-L1 expression is associated with epithelial-to-mesenchymal transition in adenocarcinoma of the lung. Human pathology. 2016;58:714.

32. Chen L, Xiong Y, Li J, Zheng X, Zhou Q, Turner A, et al. PD-L1 expression promotes epithelial 
to mesenchymal transition in human esophageal cancer. Cellular physiology biochemistry. $2017 ; 42: 2267-80$

33. Qiu XY, Hu DX, Chen W-Q, Chen RQ, Qian SR, Li CY, et al. PD-L1 confers glioblastoma multiforme malignancy via Ras binding and Ras/Erk/EMT activation. BBA-Molecular Basis of Disease. 2018;1864:1754-69.

34. Yasutoshi A, Akemi K, Hiroyuki N, Yasumasa I, Takeshi T, Hideo Y, et al. Expression of the PD-1 antigen on the surface of stimulated mouse T and B lymphocytes. International Immunology. 1996:5.

35. Jr BD, Bakan CA, Hofmeister CC, Efebera Y, Becknell B, Baiocchi RA, et al. The PD-1/PDL1 axis modulates the natural killer cell versus multiple myeloma effect: a therapeutic target for CT011, a novel monoclonal anti-PD-1 antibody. blood. 2010;116:2286-94.

36. Karyampudi L, Lamichhane P, Krempski J, Kalli KR, Behrens MD, Vargas DM, et al. PD-1 Blunts the Function of Ovarian Tumor-Infiltrating Dendritic Cells by Inactivating NF-кB. Cancer Research. 2016;76:239.

37. Gordon SR, Maute RL, Dulken BW, Hutter G, George BM, McCracken MN, et al. PD-1 expression by tumour-associated macrophages inhibits phagocytosis and tumour immunity. Nature. 2017;7655:495-9.

38. Lee Y, Shin JH, Longmire M, Wang H, Kohrt HE, Chang HY, et al. CD44+ cells in head and neck squamous cell carcinoma suppress T-cell-mediated immunity by selective constitutive and inducible expression of PD-L1. Clinical Cancer Research. 2016;22:3571-81.

39. Vassilakopoulou M, Avgeris M, Velcheti V, Kotoula V, Rampias T, Chatzopoulos K, et al. Evaluation of PD-L1 expression and associated tumor-infiltrating lymphocytes in laryngeal squamous cell carcinoma. Clinical Cancer Research. 2016;22:704-13.

40. Rey O, Yuan J, Young SH, Rozengurt E. Protein kinase Cv/protein kinase D3 nuclear localization, catalytic activation, and intracellular redistribution in response to $G$ protein-coupled receptor agonists. Journal of Biological Chemistry. 2003;278:23773-85.

41. Wetzker R, Böhmer F-D. Transactivation joins multiple tracks to the ERK/MAPK cascade. Nature reviews Molecular cell biology. 2003;4:651-7.

42. Burger M, Hartmann T, Burger JA, Schraufstatter I. KSHV-GPCR and CXCR2 transforming capacity and angiogenic responses are mediated through a JAK2-STAT3-dependent pathway. 
Oncogene. 2005;24:2067-75.

43. Wang Y, Lu X, Zhu L, Shen Y, Chengedza S, Feng H, et al. IKK epsilon kinase is crucial for viral G protein-coupled receptor tumorigenesis. Proceedings of the National Academy of Sciences. 2013;110:11139-44.

44. BUGGY, Joseph?J. Binding of $\alpha$-melanocyte-stimulating hormone to its G-protein-coupled receptor on B-lymphocytes activates the Jak/STAT pathway. Biochemical Journal. 1998;331:211-6. 45. Chen R-J, Ho Y-S, Guo H-R, Wang Y-J. Rapid activation of Stat3 and ERK1/2 by nicotine modulates cell proliferation in human bladder cancer cells. Toxicological Sciences. 2008;104:28393.

46. Plaza-Menacho I, van der Sluis T, Hollema H, Gimm O, Buys CH, Magee AI, et al. Ras/ERK1/2-mediated STAT3 Ser727 phosphorylation by familial medullary thyroid carcinomaassociated RET mutants induces full activation of STAT3 and is required for c-fos promoter activation, cell mitogenicity, and transformation. Journal of Biological Chemistry. 2007;282:641524.

47. Sun Q, Liu L, Mandal J, Molino A, Stolz D, Tamm M, et al. PDGF-BB induces PRMT1 expression through ERK1/2 dependent STAT1 activation and regulates remodeling in primary human lung fibroblasts. Cellular signalling. 2016;28:307-15.

48. Cho HJ, Kim SK, Jin SM, Hwang EM, Kim YS, Huh K, et al. IFN- $\gamma$-induced BACE1 expression is mediated by activation of JAK 2 and ERK1/2 signaling pathways and direct binding of STAT1 to BACE1 promoter in astrocytes. Glia. 2007;55:253-62.

49. Gough DJ, Koetz L, Levy DEJPo. The MEK-ERK pathway is necessary for serine phosphorylation of mitochondrial STAT3 and Ras-mediated transformation. PloS one. 2013;8.

50. GKOUvERIS I, Nikitakis N, Karanikou M, Rassidakis G, SKLAvOUNOU AJOr. Erk1/2 activation and modulation of STAT3 signaling in oral cancer. Oncology reports. 2014;32:2175-82. 51. Fang Y, Zhong L, Lin M, Zhou X, Jing H, Ying M, et al. MEK/ERK dependent activation of STAT1 mediates dasatinib-induced differentiation of acute myeloid leukemia. PloS one. 2013;8.

\section{FIGURE LEGENDS}

Fig. 1 Expression of PKD3 in OSCC and its relationship with EMT. a The expression levels of PKD3 and PD-L1 were analyzed by TCGA-HNSCC. b Correlation analysis was performed using 
the expression data of PKD3, PD-L1, and EMT markers in HNSCC from TCGA. c, d The expression of PKD3, PD-L1, and EMT markers was detected by western blot in OSCC cells and non-tumor cells of oral epithelium. Densitometry was analyzed, and the correlation between PKD3, PD-L1, and EMT marker proteins was determined. e Expression of PKD3, PD-L1, and EMT markers in OSCC tissues detected by immunohistochemistry. f TCGA database was used to analyze the levels of PKD3 and Vimentin in HNSCC of different grades. $* * * \mathrm{P}<0.001$.

Fig. 2 PKD3 down-regulation suppresses the proliferation, migration, and invasion of OSCC cells. a, $\mathbf{b}$ Effects of PKD3 on the proliferation of Cal-27 and HSC-4 cells evaluated using clone formation and CCK-8 assays. c Transwell migration assays performed with Cal-27 and HSC-4 cells transfected with shRNA-PKD3 or shRNA-Con plasmids. The number of migrated cells per field is shown in the bar graphs (right panel). d Wound-healing assay with Cal-27 and HSC-4 cells transfected with shRNA-PKD3 or shRNA-Con plasmid. Images were captured at 0,12 , and $24 \mathrm{~h}$ after inducing the wound. e Transwell invasion assay for the indicated cell lines. The number of cells passing through the Matrigel per field is shown in the bar graphs (right panel). f Expression of PD-L1 and EMT markers in Cal-27 and HSC-4 cells transfected with shRNA-PKD3 or shRNA-Con, detected by western blot. All experiments were repeated three times. Data are presented as the mean $\pm \mathrm{SD} . * * \mathrm{P}$ $<0.01 ; * * * \mathrm{P}<0.001$.

Fig. 3 PKD3 overexpression can promote the proliferation, migration, and invasion of OSCC cells. a Colony formation of DOK and Cal-27 cells after PKD3 overexpression. b Growth curves. c Transwell migration assay with DOK and Cal-27 cells transfected with pCDNA3.1/NT-PKD3 (OEPKD3) or control plasmid (Con). The number of migrated cells per field is shown in the bar graphs (right panel). d Wound-healing assay with PKD3-overexpressing and control cells. Images were captured at 0,12 , and $24 \mathrm{~h}$ post wound. e Transwell invasion assay for the indicated cell lines. The number of invading cells per field is shown in the bar graphs (right panel). $\mathbf{f}$ Western blot analysis of PD-L1 and EMT markers in DOK and Cal-27 cells transfected with pCDNA3.1/NT-PKD3 (OEPKD3) or control plasmid (Con). All experiments shown are representative of three independent experiments. Data are presented as the mean $\pm \mathrm{SD} .{ }^{*} \mathrm{P}<0.05 ; * * \mathrm{P}<0.01 ; * * * \mathrm{P}<0.001$.

Fig. 4 The role of PKD3 in the EMT phenotype of OSCC induced by the PD-1/PD-L1 pathway. a, 
b Western blot was used to detect the levels of PKD3, PD-L1, and EMT markers in Cal-27 cells treated with PD-1 fusion protein. $\mathbf{c}, \mathbf{d}$ Protein levels of PKD3, PD-L1, and EMT markers after stable knockdown of PKD3 in Cal-27 cells treated with or without PD-1 fusion protein. ${ }^{*} \mathrm{P}<0.05$; ${ }^{*} \mathrm{P}<$ $0.01 ; * * * \mathrm{P}<0.001$.

Fig. 5 Both PKD3 and PD-L1 activate ERK1/2 and STAT1/3 pathways to promote EMT in OSCC cells. a Activation of EMT-related factors and expression of EMT markers after transfecting PKD3 and/or PD-L1 siRNAs in Cal-27, analyzed by western blot. b Quantification of PKD3, PD-L1, and EMT markers by densitometry analysis. c Densitometric analysis of changes in the abundance of phosphorylated substrates and total proteins, normalized to GAPDH as a loading control. ***P $<$ 0.001 .

Fig. 6 ERK1/2 and STAT1/3 pathways modulate PKD3 and PD-L1 expression and regulate EMT in OSCC cells. a-c PKD3, PD-L1, and EMT were detected by western blot after transfection of ERK1/2, STAT1, and STAT3 siRNAs into Cal-27 cells treated with or without PD-1 fusion protein. d Western blot analysis of ERK1/2 phosphorylation and STAT1/3 phosphorylation at S727, after transfecting ERK1/2 siRNA in Cal-27 cells treated with or without PD-1 fusion protein.

Fig. 7 PKD3 promotes the growth and lymph node metastasis of OSCC cells in vivo. a The xenograft tumor metastasis model was established by inoculating cells subcutaneously in the footpad of nude mice. $\mathbf{b}, \mathbf{d}$ Nude mouse footpads were inoculated with Cal-27 Con, sh-PKD3-1, and sh-PKD3-2 cells. Four weeks later, subcutaneous tumors of the footpads and left popliteal lymph nodes were taken, and their volume was measured. $\mathbf{c}$ Immunohistochemical staining of PKD3 and PD-L1 in subcutaneous footpad tumors. e Immunohistochemical staining of GFP was used to evaluate the ratio of metastatic lymph nodes among different groups. ${ }^{* *} \mathrm{P}<0.01 ; * * * \mathrm{P}<0.001$.

Fig. 8 The prediction model of PKD3/PD-L1 positive feedback loop. PKD3/PD-L1 drive EMT through the ERK/STAT1/3 signaling, thus promoting tumor growth and metastasis.

Figure S1 TCGA gene expression database was used to analyze the expression of PKD3 across different ages, sexes, and races. 



\section{Figures}

Fig.1
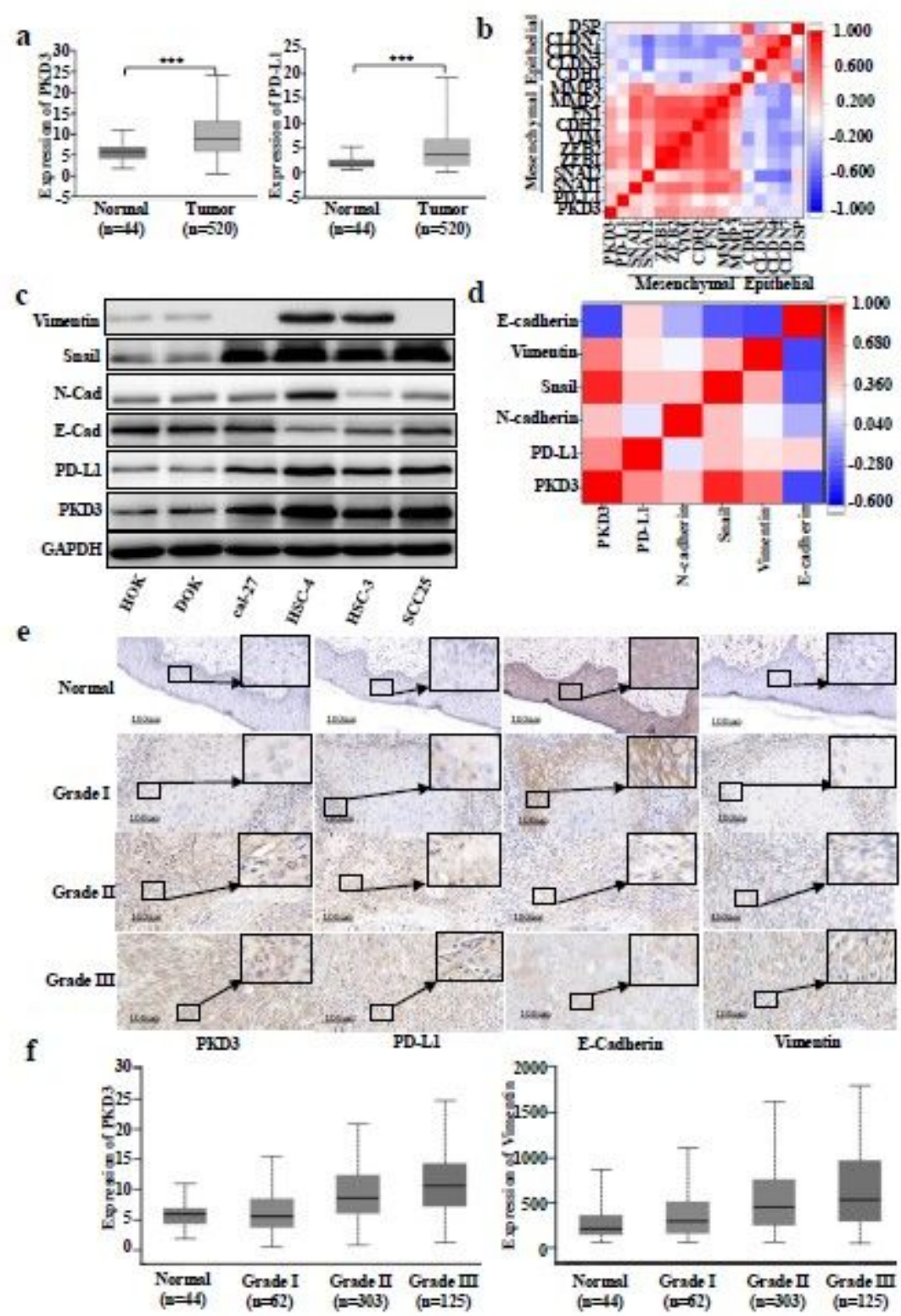

\section{Figure 1}

Expression of PKD3 in OSCC and its relationship with EMT. a The expression levels of PKD3 and PD-L1 were analyzed by TCGA-HNSCC. $b$ Correlation analysis was performed using the expression data of PKD3, PD-L1, and EMT markers in HNSCC from TCGA. c, d The expression of PKD3, PD-L1, and EMT markers was detected by western blot in OSCC cells and non-tumor cells of oral epithelium. Densitometry was analyzed, and the correlation between PKD3, PD-L1, and EMT marker proteins was determined. e Expression of PKD3, PD-L1, and EMT markers in OSCC tissues detected by immunohistochemistry. $f$ 
TCGA database was used to analyze the levels of PKD3 and Vimentin in HNSCC of different grades. $* \star * P$ $<0.001$.

\section{Fig. 2}

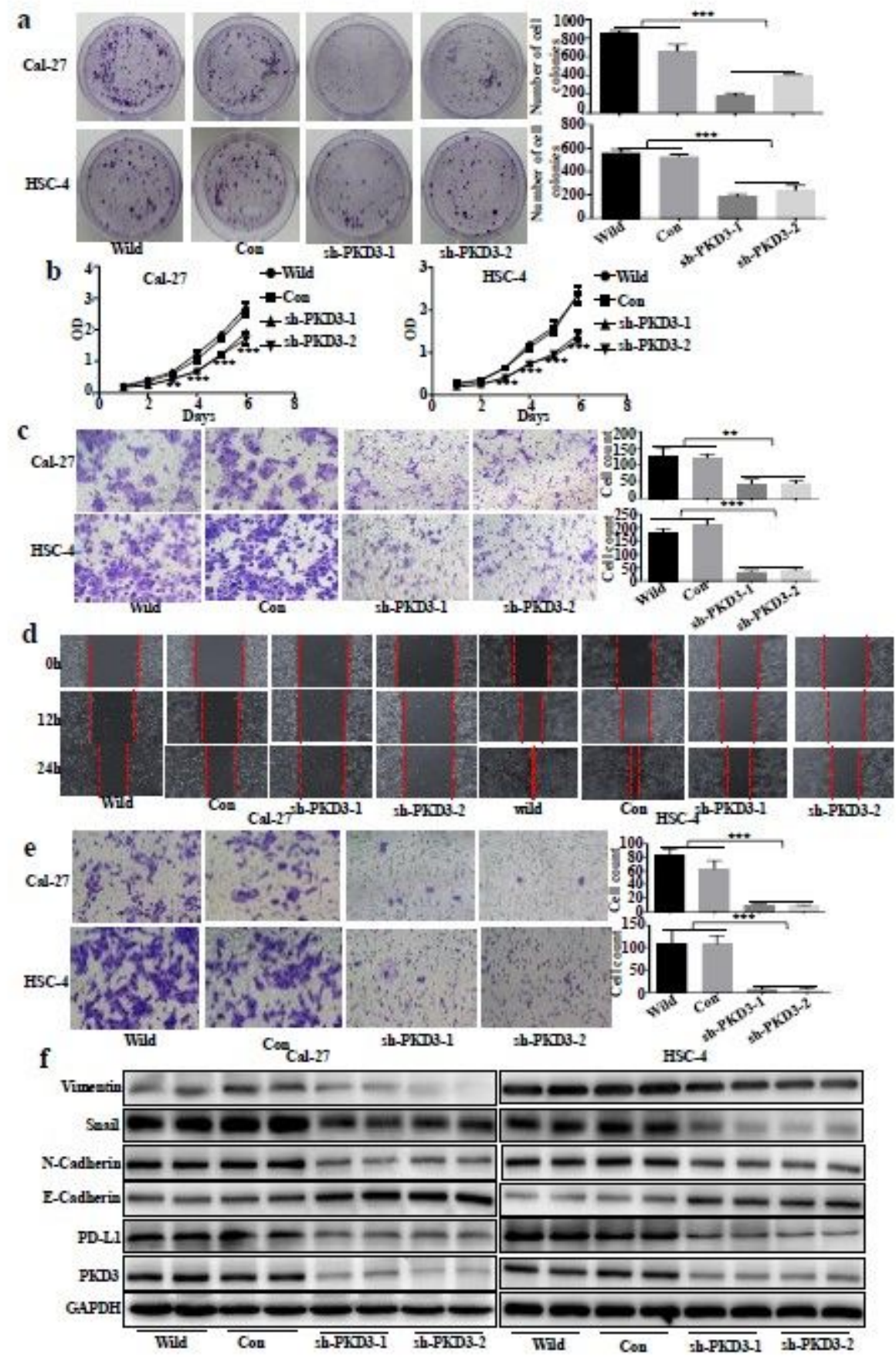

Figure 2

PKD3 down-regulation suppresses the proliferation, migration, and invasion of OSCC cells. a, b Effects of PKD3 on the proliferation of Cal-27 and HSC-4 cells evaluated using clone formation and CCK-8 assays. C Transwell migration assays performed with Cal-27 and HSC-4 cells transfected with shRNA-PKD3 or shRNA-Con plasmids. The number of migrated cells per field is shown in the bar graphs (right panel). $d$ 
Wound-healing assay with Cal-27 and HSC-4 cells transfected with shRNA-PKD3 or shRNA-Con plasmid. Images were captured at 0,12, and $24 \mathrm{~h}$ after inducing the wound. e Transwell invasion assay for the indicated cell lines. The number of cells passing through the Matrigel per field is shown in the bar graphs (right panel). $\mathrm{f}$ Expression of PD-L1 and EMT markers in Cal-27 and HSC-4 cells transfected with shRNAPKD3 or shRNA-Con, detected by western blot. All experiments were repeated three times. Data are presented as the mean \pm SD. $* * P<0.01 ; * \star * P<0.001$.

Fig.3

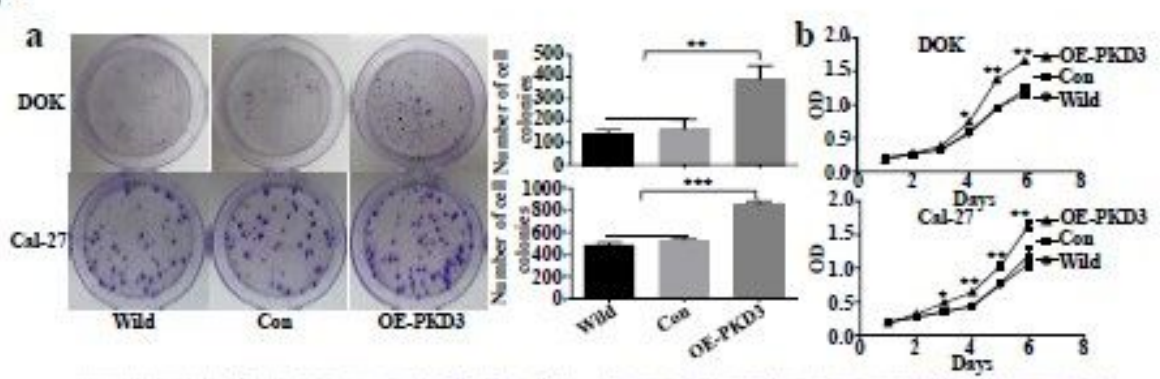

c
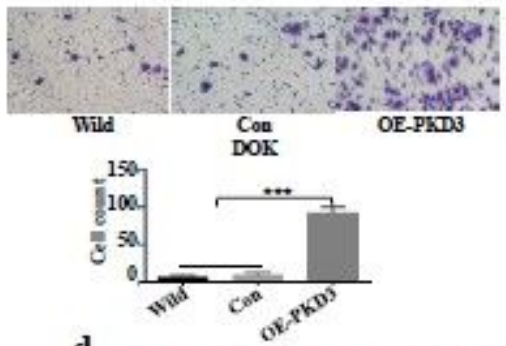

d
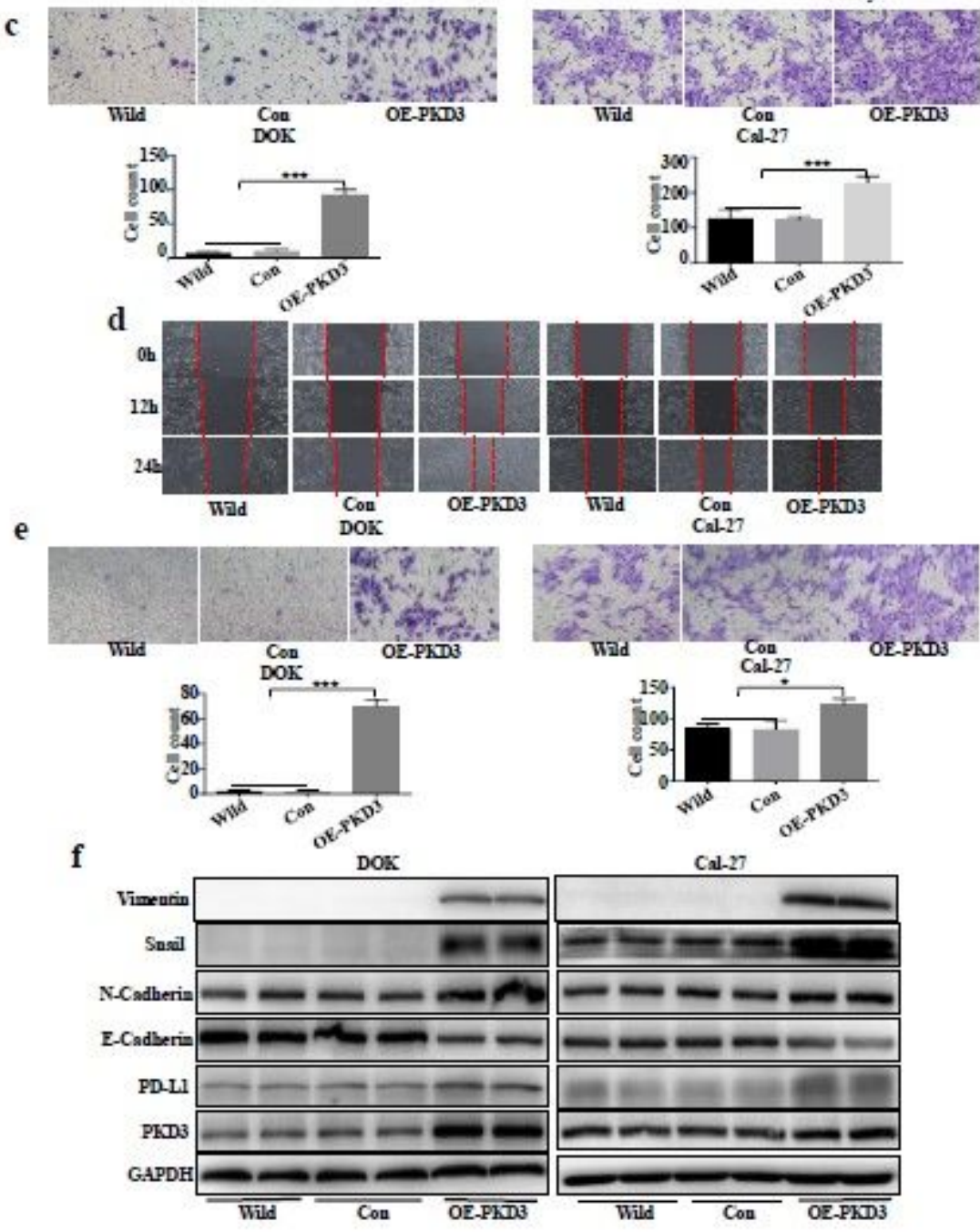

e
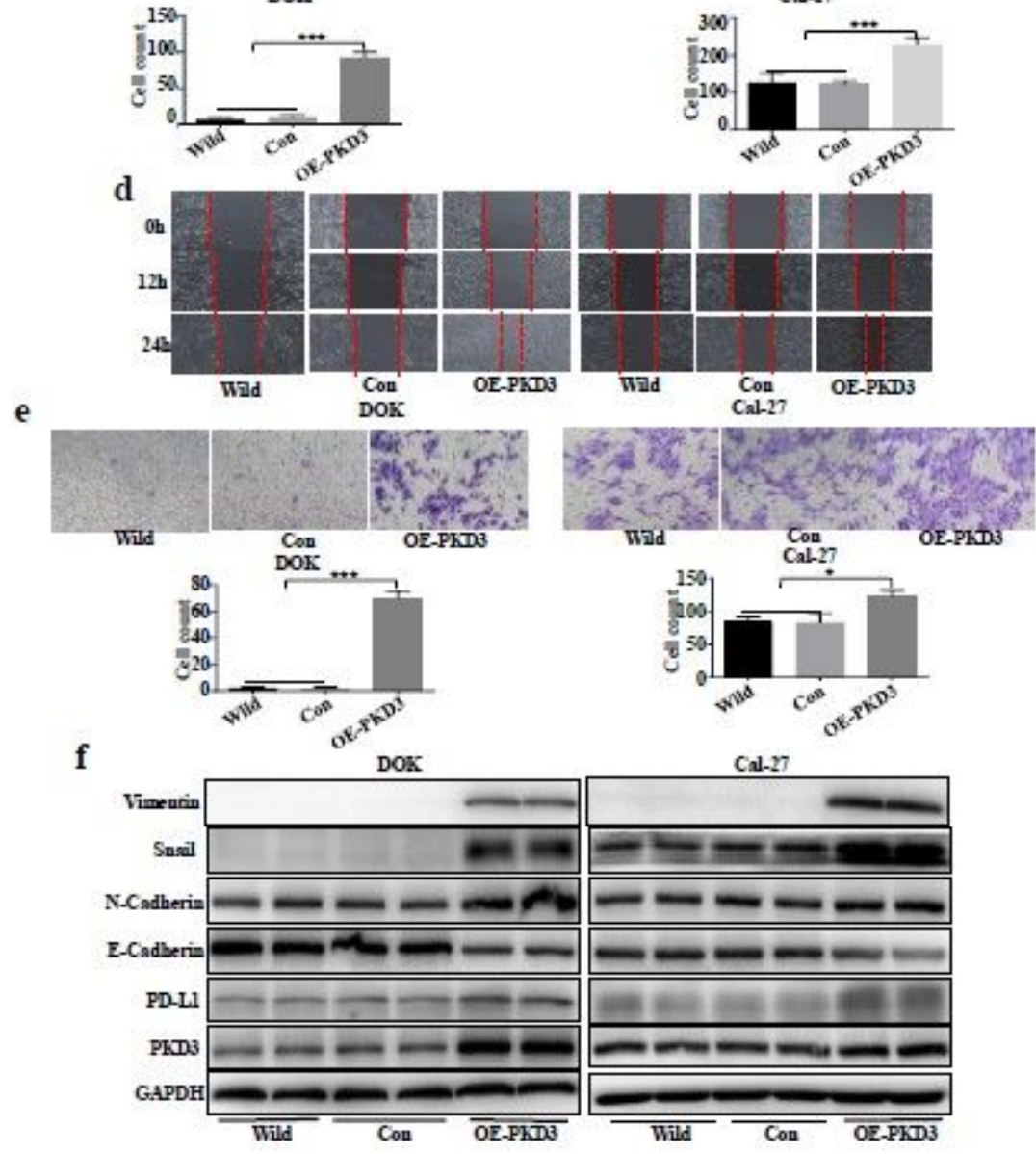

Figure 3 
PKD3 overexpression can promote the proliferation, migration, and invasion of OSCC cells. a Colony formation of DOK and Cal-27 cells after PKD3 overexpression. b Growth curves. c Transwell migration assay with DOK and Cal-27 cells transfected with pCDNA3.1/NT-PKD3 (OE-PKD3) or control plasmid (Con). The number of migrated cells per field is shown in the bar graphs (right panel). $d$ Wound-healing assay with PKD3-overexpressing and control cells. Images were captured at 0,12 , and $24 \mathrm{~h}$ post wound. e Transwell invasion assay for the indicated cell lines. The number of invading cells per field is shown in the bar graphs (right panel). $f$ Western blot analysis of PD-L1 and EMT markers in DOK and Cal-27 cells transfected with pCDNA3.1/NT-PKD3 (OE-PKD3) or control plasmid (Con). All experiments shown are representative of three independent experiments. Data are presented as the mean $\pm S D$. ${ }^{\star} P<0.05$; $* * P<$ $0.01 ; * \star * \mathrm{P}<0.001$. 
Fig.4
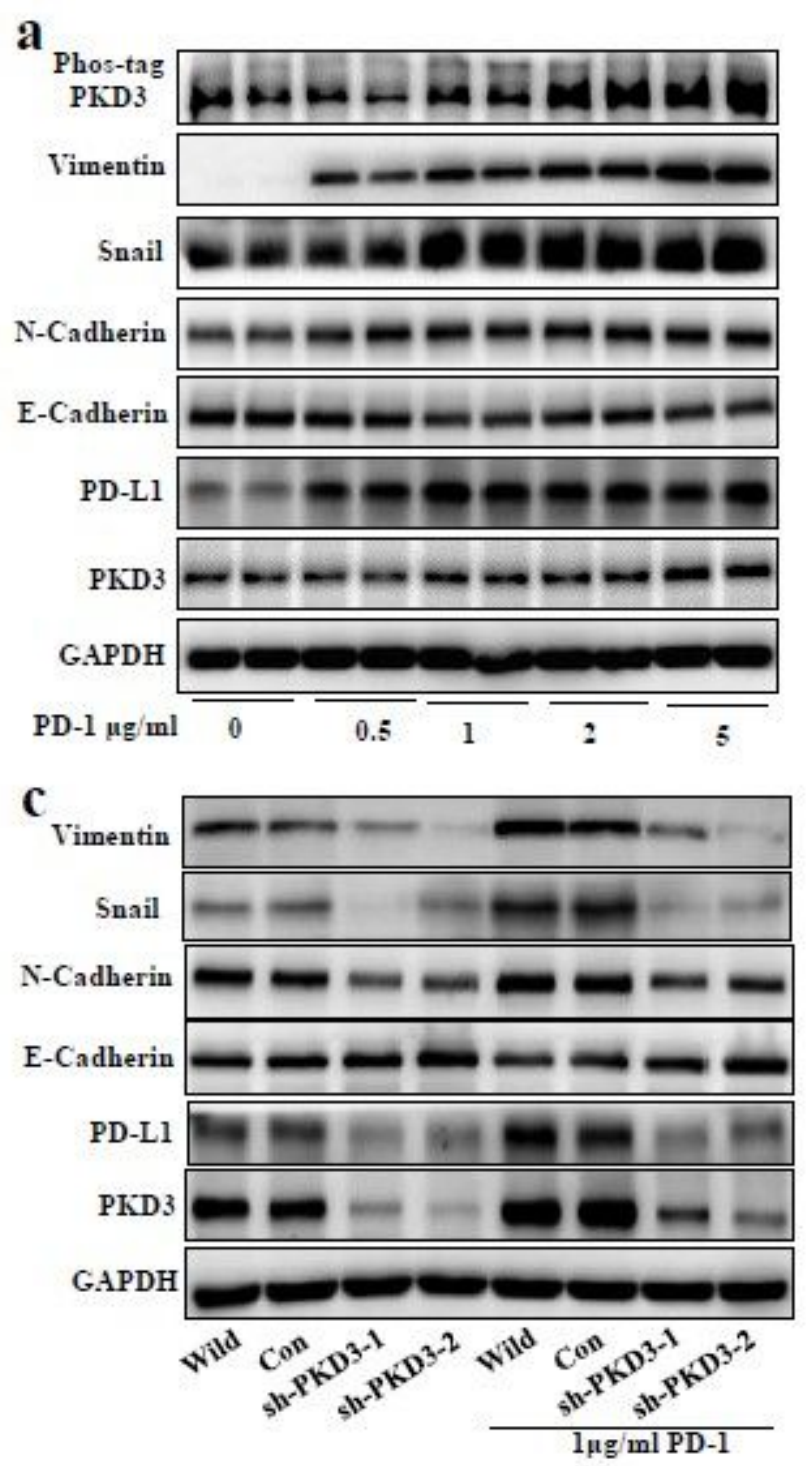

b
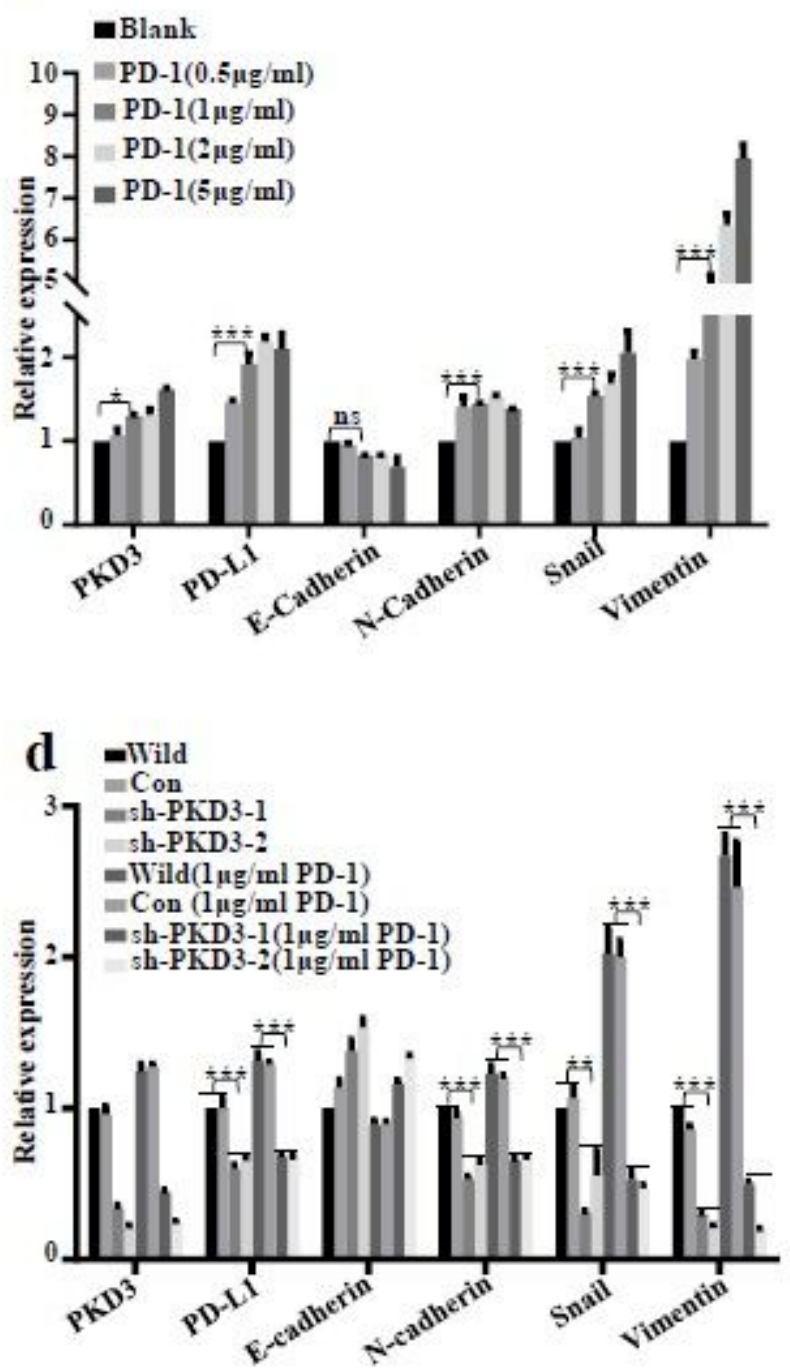

\section{Figure 4}

The role of PKD3 in the EMT phenotype of OSCC induced by the PD-1/PD-L1 pathway. a, b Western blot was used to detect the levels of PKD3, PD-L1, and EMT markers in Cal-27 cells treated with PD-1 fusion protein. c, d Protein levels of PKD3, PD-L1, and EMT markers after stable knockdown of PKD3 in Cal-27 cells treated with or without PD-1 fusion protein. ${ }^{*} \mathrm{P}<0.05$; ${ }^{\star \star} \mathrm{P}<0.01 ; \star \star \star \mathrm{P}<0.001$. 
Fig.5

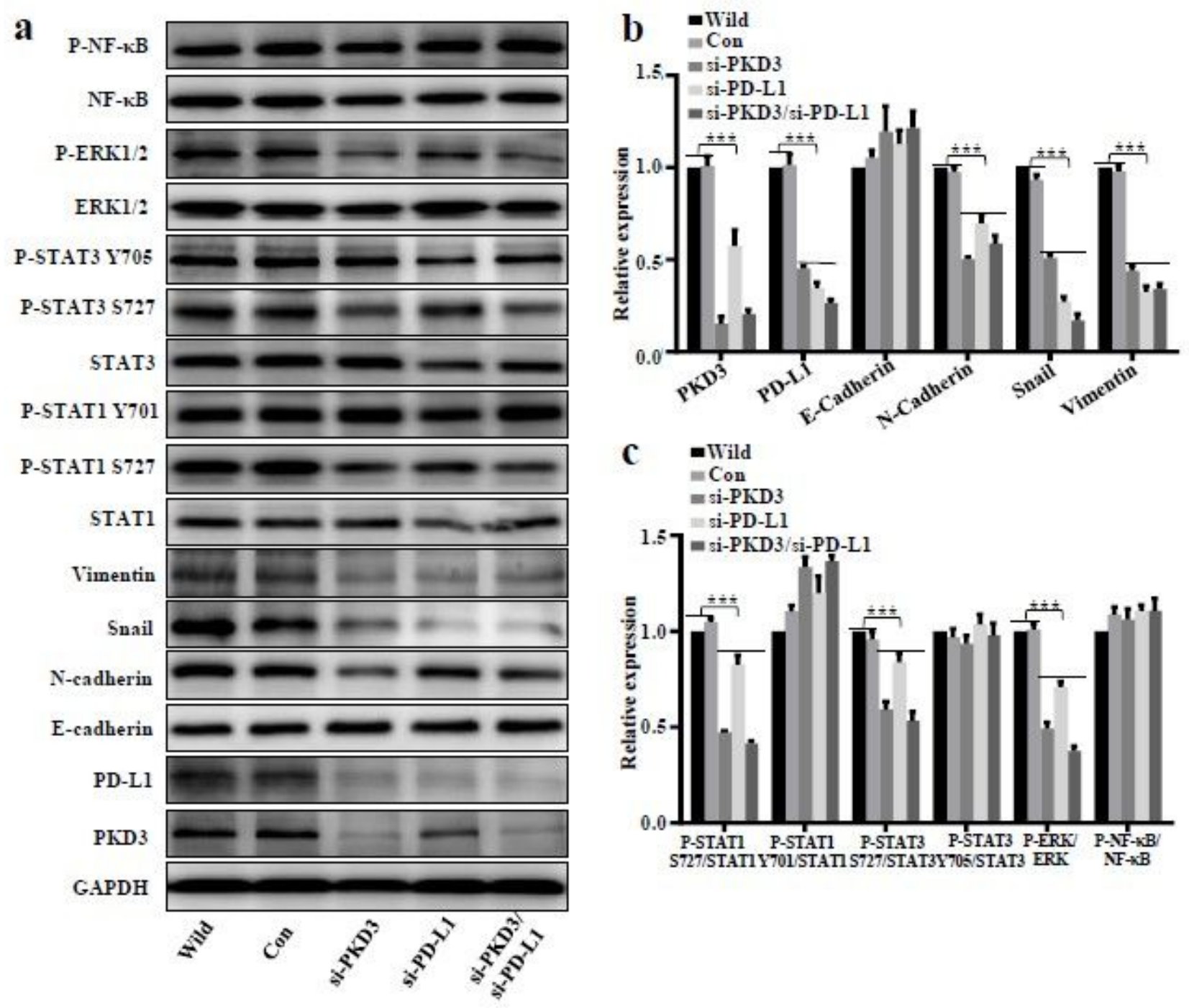

Figure 5

Both PKD3 and PD-L1 activate ERK1/2 and STAT1/3 pathways to promote EMT in OSCC cells. a Activation of EMT-related factors and expression of EMT markers after transfecting PKD3 and/or PD-L1 siRNAs in Cal-27, analyzed by western blot. b Quantification of PKD3, PD-L1, and EMT markers by densitometry analysis. c Densitometric analysis of changes in the abundance of phosphorylated substrates and total proteins, normalized to GAPDH as a loading control. $* * * P<0.001$. 


\section{Fig.6}

a

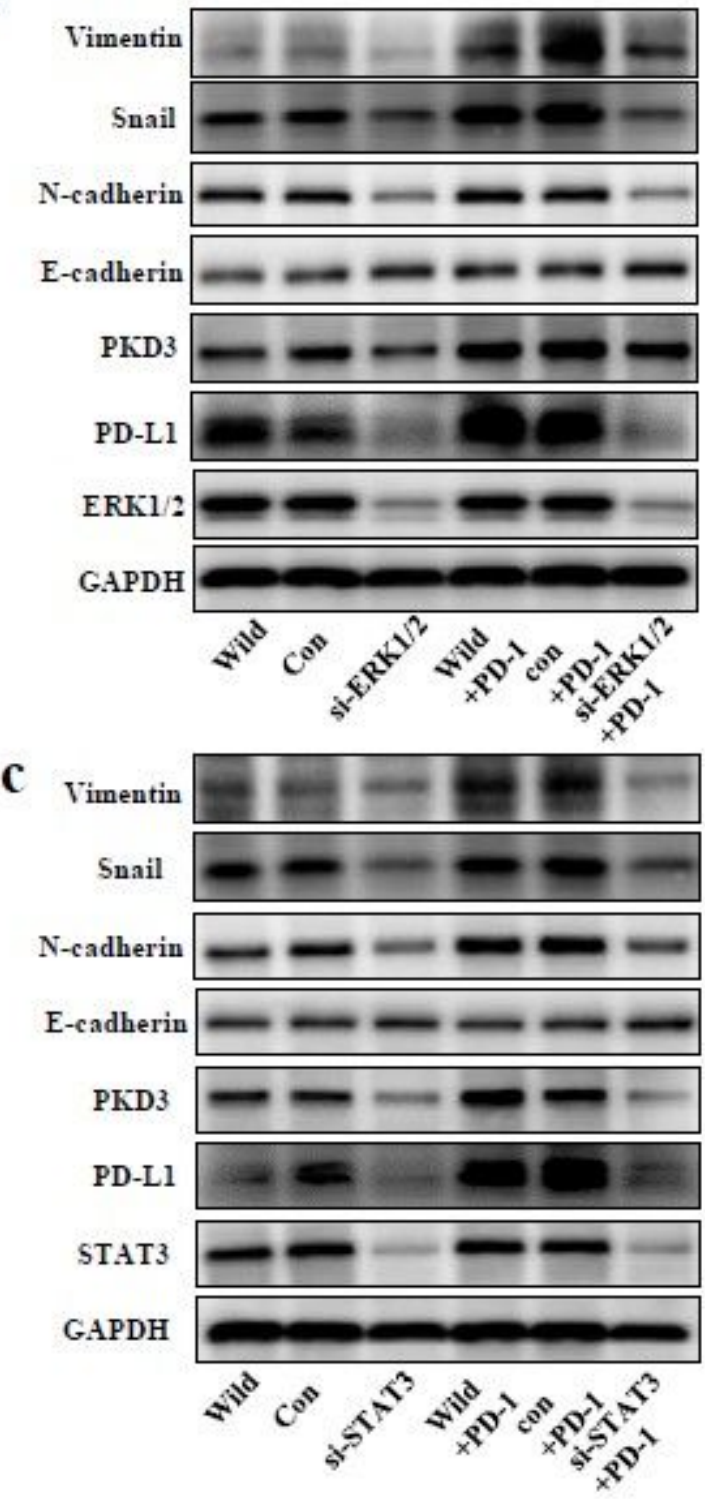

b

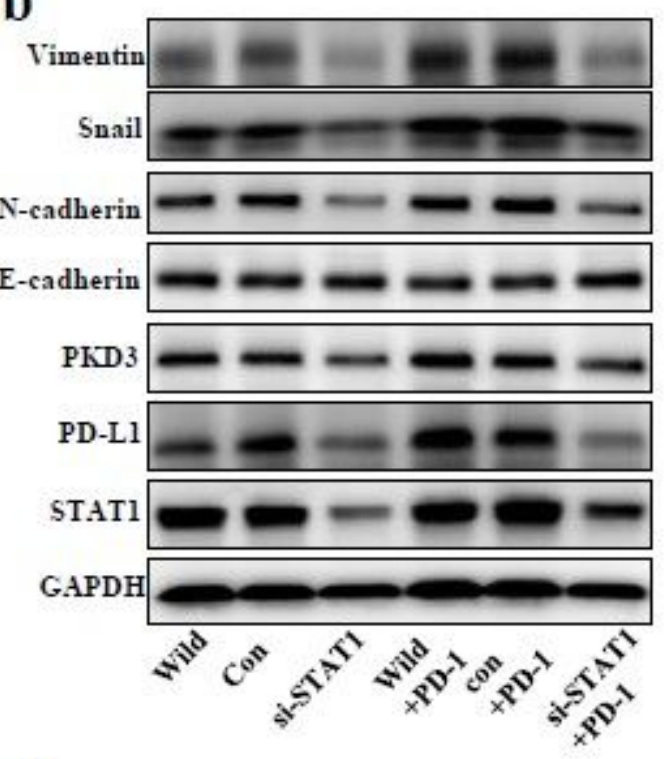

d

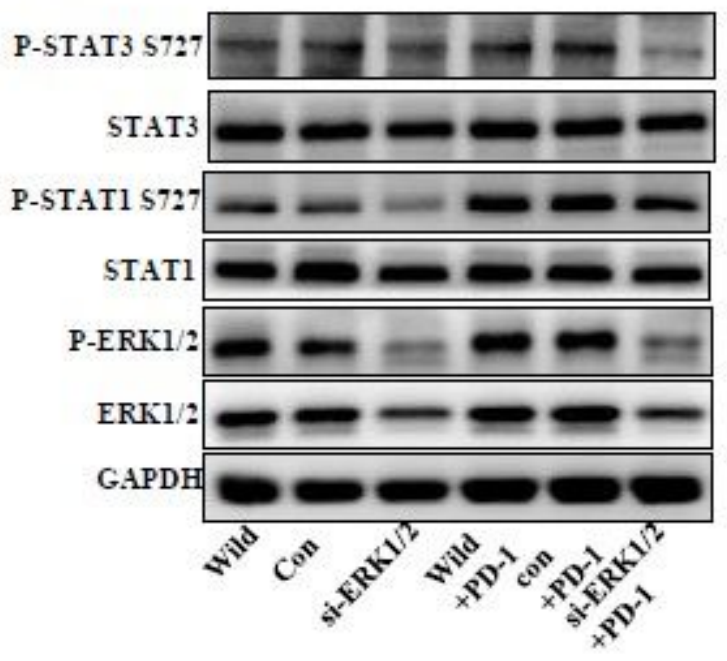

Figure 6

ERK1/2 and STAT1/3 pathways modulate PKD3 and PD-L1 expression and regulate EMT in OSCC cells. a-c PKD3, PD-L1, and EMT were detected by western blot after transfection of ERK1/2, STAT1, and STAT3 siRNAs into Cal-27 cells treated with or without PD-1 fusion protein. $d$ Western blot analysis of ERK1/2 phosphorylation and STAT1/3 phosphorylation at S727, after transfecting ERK1/2 siRNA in Cal-27 cells treated with or without PD-1 fusion protein. 


\section{Fig.7}

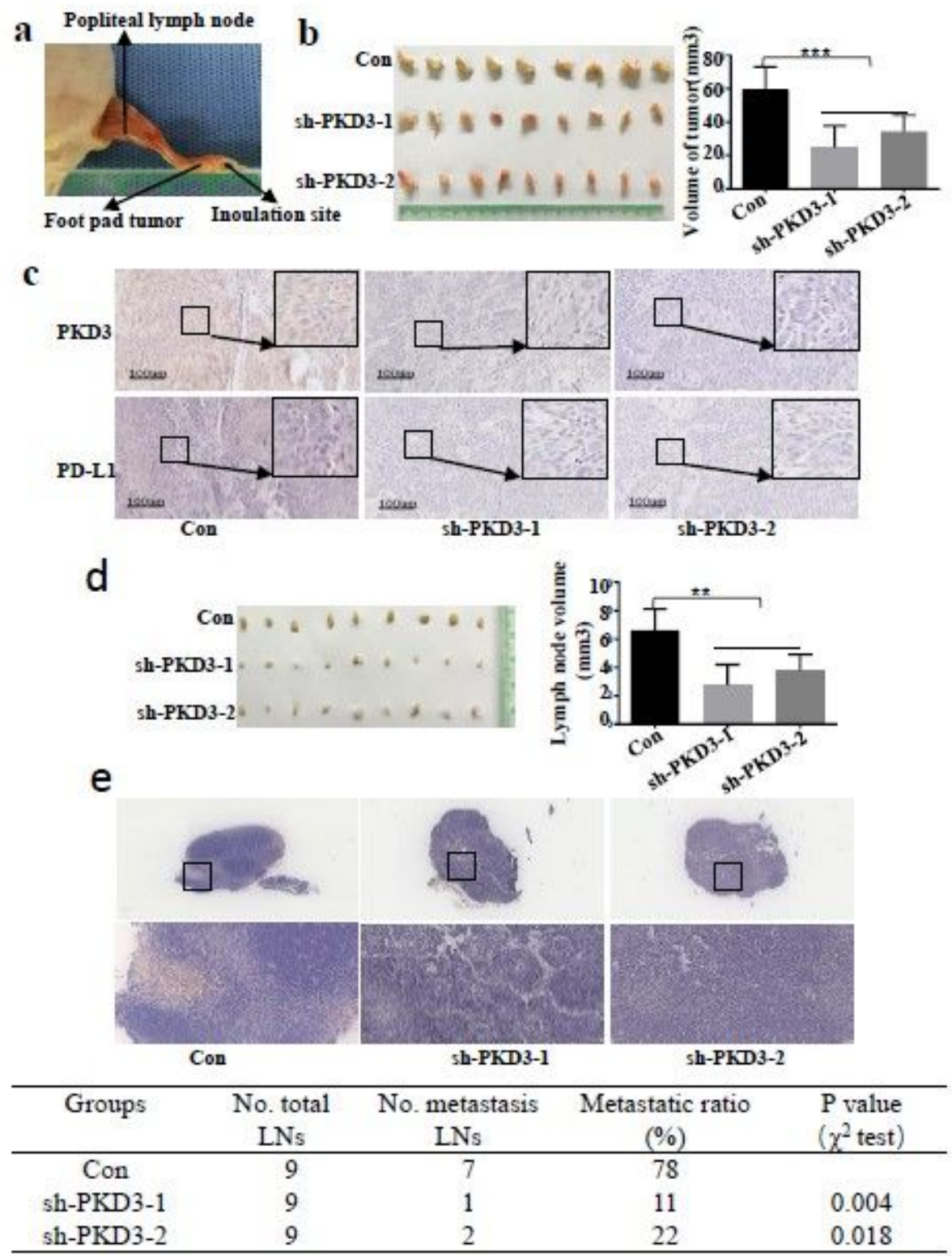

\section{Figure 7}

PKD3 promotes the growth and lymph node metastasis of OSCC cells in vivo. a The xenograft tumor metastasis model was established by inoculating cells subcutaneously in the footpad of nude mice. b, $d$ Nude mouse footpads were inoculated with Cal-27 Con, sh-PKD3-1, and sh-PKD3-2 cells. Four weeks later, subcutaneous tumors of the footpads and left popliteal lymph nodes were taken, and their volume was measured. c Immunohistochemical staining of PKD3 and PD-L1 in subcutaneous footpad tumors. e 
Immunohistochemical staining of GFP was used to evaluate the ratio of metastatic lymph nodes among different groups. ${ }^{\star \star} \mathrm{P}<0.01 ; * \star \star \mathrm{P}<0.001$.

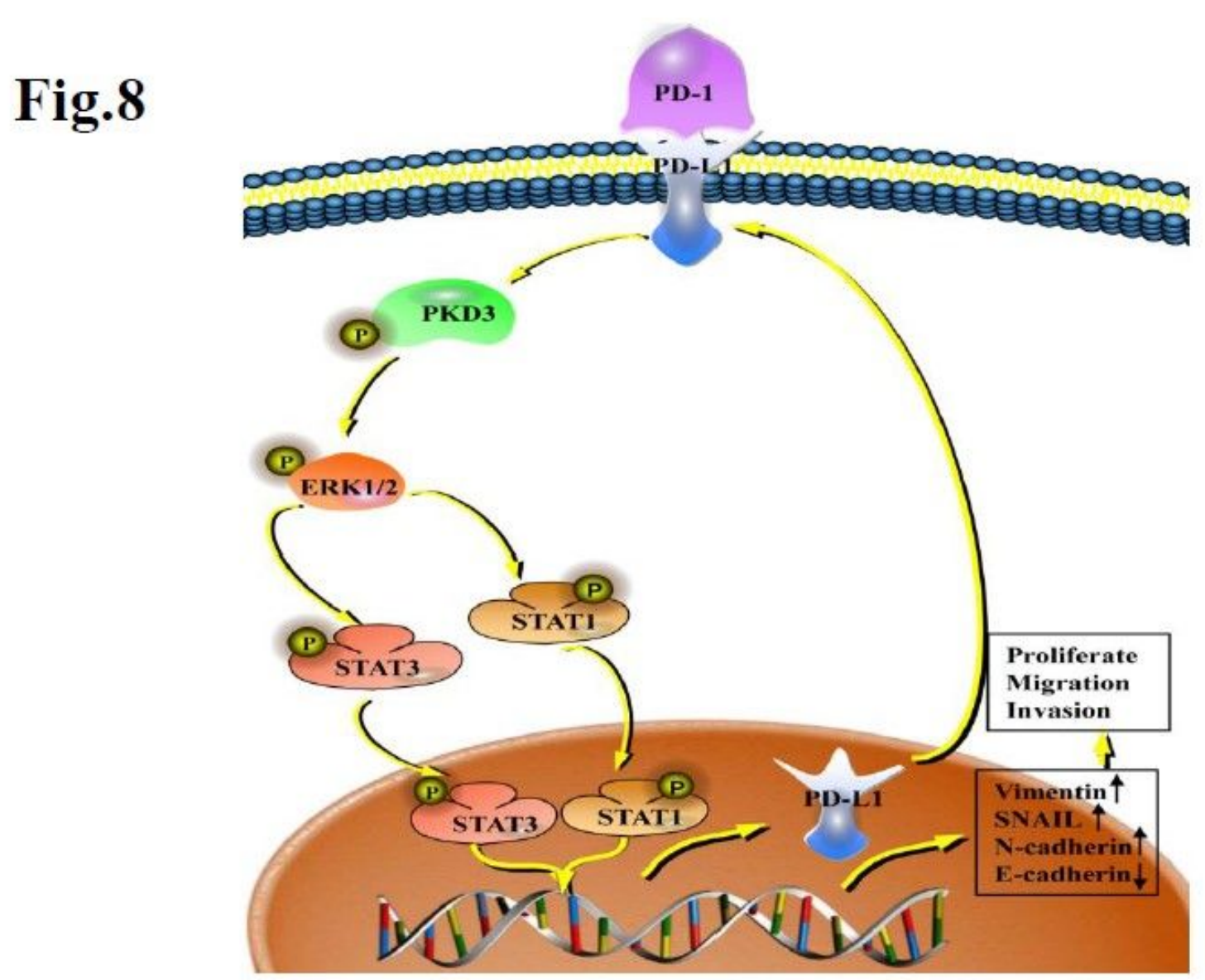

Figure 8

The prediction model of PKD3/PD-L1 positive feedback loop. PKD3/PD-L1 drive EMT through the ERK/STAT1/3 signaling, thus promoting tumor growth and metastasis.

\section{Supplementary Files}

This is a list of supplementary files associated with this preprint. Click to download.

- Additionalfile3.pdf

- Additionalfile2.pdf

- Additionalfile1.pdf

- Additionalfile4.pdf 